\title{
Pacific
}

Journal of

Mathematics

\section{BOTANY OF IRREDUCIBLE AUTOMORPHISMS OF FREE GROUPS}

ThIERry COUlbois AND ARNAUd Hilion

Volume $256 \quad$ No. 2

April 2012 


\title{
BOTANY OF IRREDUCIBLE AUTOMORPHISMS OF FREE GROUPS
}

\author{
ThIERry COUlbois AND ARnAud Hilion
}

\begin{abstract}
We give a classification of iwip (i.e., fully irreducible) outer automorphisms of the free group, by discussing the properties of their attracting and repelling trees.
\end{abstract}

\section{Introduction}

An outer automorphism $\Phi$ of the free group $F_{N}$ is fully irreducible (abbreviated as iwip) if no positive power $\Phi^{n}$ fixes a proper free factor of $F_{N}$. Being an iwip is one (in fact the most important) of the analogs for free groups of being pseudo-Anosov for mapping classes of hyperbolic surfaces. Another analog of pseudo-Anosov is the notion of an atoroidal automorphism: an element $\Phi \in \operatorname{Out}\left(F_{N}\right)$ is atoroidal or hyperbolic if no positive power $\Phi^{n}$ fixes a nontrivial conjugacy class. Bestvina and Feighn [1992] and Brinkmann [2000] proved that $\Phi$ is atoroidal if and only if the mapping torus $F_{N} \rtimes_{\Phi} \mathbb{Z}$ is Gromov-hyperbolic.

Pseudo-Anosov mapping classes are known to be "generic" elements of the mapping class group (in various senses). Rivin [2008] and Sisto [2011] recently proved that, in the sense of random walks, generic elements of $\operatorname{Out}\left(F_{N}\right)$ are atoroidal iwip automorphisms.

Bestvina and Handel [1992] proved that iwip automorphisms have the key property of being represented by (absolute) train-track maps.

A pseudo-Anosov element $f$ fixes two projective classes of measured foliations $\left[\left(\mathscr{F}^{+}, \mu^{+}\right)\right]$and $\left[\left(\mathscr{F}^{-}, \mu^{-}\right)\right]$:

$$
\left(\mathscr{F}^{+}, \mu^{+}\right) \cdot f=\left(\mathscr{F}^{+}, \lambda \mu^{+}\right) \text {and }\left(\mathscr{F}^{-}, \mu^{-}\right) \cdot f=\left(\mathscr{F}^{-}, \lambda^{-1} \mu^{-}\right),
$$

where $\lambda>1$ is the expansion factor of $f$. Alternatively, considering the dual $\mathbb{R}$ trees $T^{+}$and $T^{-}$, we get:

$$
T^{+} \cdot f=\lambda T^{+} \quad \text { and } \quad T^{-} \cdot f=\lambda^{-1} T^{-} .
$$

We now discuss the analogous situation for iwip automorphisms. The group of outer automorphisms $\operatorname{Out}\left(F_{N}\right)$ acts on the outer space $\mathrm{CV}_{N}$ and its boundary

MSC2010: 20E05, 20E08, 20F65, 57R30.

Keywords: free group automorphisms, real trees, laminations, iwip. 
$\partial \mathrm{CV}_{N}$. Recall that the compactified outer space $\overline{\mathrm{CV}}_{N}=\mathrm{CV}_{N} \cup \partial \mathrm{CV}_{N}$ is made up of (projective classes of) $\mathbb{R}$-trees with an action of $F_{N}$ by isometries which is minimal and very small. See [Vogtmann 2002] for a survey on outer space. An iwip outer automorphism $\Phi$ has north-south dynamics on $\overline{\mathrm{CV}}_{N}$ : it has a unique attracting fixed tree $\left[T_{\Phi}\right]$ and a unique repelling fixed tree $\left[T_{\Phi^{-1}}\right]$ in the boundary of outer space (see [Levitt and Lustig 2003]):

$$
T_{\Phi} \cdot \Phi=\lambda_{\Phi} T_{\Phi} \text { and } T_{\Phi^{-1}} \cdot \Phi=\frac{1}{\lambda_{\Phi^{-1}}} T_{\Phi^{-1}},
$$

where $\lambda_{\Phi}>1$ is the expansion factor of $\Phi$ (i.e., the exponential growth rate of nonperiodic conjugacy classes).

Contrary to the pseudo-Anosov setting, the expansion factor $\lambda_{\Phi}$ of $\Phi$ is typically different from the expansion factor $\lambda_{\Phi^{-1}}$ of $\Phi^{-1}$. More generally, qualitative properties of the fixed trees $T_{\Phi}$ and $T_{\Phi^{-1}}$ can be fairly different. This is the purpose of this paper to discuss and compare the properties of $\Phi, T_{\Phi}$ and $T_{\Phi^{-1}}$.

First, the free group, $F_{N}$, may be realized as the fundamental group of a surface $S$ with boundary. It is part of folklore that, if $\Phi$ comes from a pseudo-Anosov mapping class on $S$, then its limit trees $T_{\Phi}$ and $T_{\Phi^{-1}}$ live in the Thurston boundary of Teichmüller space: they are dual to a measured foliation on the surface. Such trees $T_{\Phi}$ and $T_{\Phi^{-1}}$ are called surface trees and such an iwip outer automorphism $\Phi$ is called geometric (in this case $S$ has exactly one boundary component).

The notion of surface trees has been generalized (see for instance [Bestvina 2002]). An $\mathbb{R}$-tree which is transverse to measured foliations on a finite CWcomplex is called geometric. It may fail to be a surface tree if the complex fails to be a surface.

If $\Phi$ does not come from a pseudo-Anosov mapping class and if $T_{\Phi}$ is geometric then $\Phi$ is called parageometric. For a parageometric iwip $\Phi$, Guirardel [2005] and Handel and Mosher [2007] proved that the repelling tree $T_{\Phi^{-1}}$ is not geometric. So we have that, $\Phi$ comes from a pseudo-Anosov mapping class on a surface with boundary if and only if both trees $T_{\Phi}$ and $T_{\Phi^{-1}}$ are geometric. Moreover in this case both trees are indeed surface trees.

In [Coulbois and Hilion 2010] we introduced a second dichotomy for trees in the boundary of outer space with dense orbits. For a tree $T$, we consider its limit set $\Omega \subseteq \bar{T}$ (where $\bar{T}$ is the metric completion of $T$ ). The limit set $\Omega$ consists of points of $\bar{T}$ with at least two pre-images by the map $2: \partial F_{N} \rightarrow \hat{T}=\bar{T} \cup \partial T$ introduced in [Levitt and Lustig 2003]; see Section 4A. We are interested in the two extremal cases: A tree $T$ in the boundary of outer space with dense orbits is of surface type if $T \subseteq \Omega$ and $T$ is of Levitt type if $\Omega$ is totally disconnected. As the terminology suggests, a surface tree is of surface type. Trees of Levitt type where discovered by Levitt [1993]. 
Combining together the two sets of properties, we introduced in [Coulbois and Hilion 2010] the following definitions. A tree $T$ in $\partial \mathrm{CV}_{N}$ with dense orbits is

- a surface tree if it is both geometric and of surface type;

- Levitt if it is geometric and of Levitt type;

- pseudo-surface if it is not geometric and of surface type;

- pseudo-Levitt if it is not geometric and of Levitt type

The following theorem is the main result of this paper.

Theorem 5.2. Let $\Phi$ be an iwip outer automorphism of $F_{N}$. Let $T_{\Phi}$ and $T_{\Phi^{-1}}$ be its attracting and repelling trees. Then exactly one of the following occurs

(1) The trees $T_{\Phi}$ and $T_{\Phi^{-1}}$ are surface trees. Equivalently, $\Phi$ is geometric.

(2) The tree $T_{\Phi}$ is Levitt (i.e., geometric and of Levitt type), and the tree $T_{\Phi^{-1}}$ is pseudo-surface (i.e., nongeometric and of surface type). Equivalently, $\Phi$ is parageometric.

(3) The tree $T_{\Phi^{-1}}$ is Levitt (i.e., geometric and of Levitt type), and the tree $T_{\Phi}$ is pseudo-surface (i.e., nongeometric and of surface type). Equivalently, $\Phi^{-1}$ is parageometric.

(4) The trees $T_{\Phi}$ and $T_{\Phi^{-1}}$ are pseudo-Levitt (nongeometric and of Levitt type).

Case (1) corresponds to toroidal iwips whereas cases (2), (3) and (4) corresponds to atoroidal iwips. In case (4) the automorphism $\Phi$ is called pseudo-Levitt.

Gaboriau, Jaeger, Levitt and Lustig [Gaboriau et al. 1998] introduced the notion of an index $\operatorname{ind}(\Phi)$, computed from the rank of the fixed subgroup and from the number of attracting fixed points of the automorphisms $\varphi$ in the outer class $\Phi$. Another index for a tree $T$ in $\overline{\mathrm{CV}}_{N}$ has been defined and studied by Gaboriau and Levitt [1995]; we call it the geometric index $\operatorname{ind}_{\text {geo }}(T)$. Finally in [Coulbois and Hilion 2010] we introduced and studied the 2-index $\operatorname{ind}_{2}(T)$ of an $\mathbb{R}$-tree $T$ in the boundary of outer space with dense orbits. The two indices $\operatorname{ind}_{\text {geo }}(T)$ and $\operatorname{ind}_{2}(T)$ describe qualitative properties of the tree $T$ [Coulbois and Hilion 2010]. We define these indices and recall our botanical classification of trees in Section 4A.

The key to prove Theorem 5.2 is this:

Propositions 4.2 and 4.4. Let $\Phi$ be an iwip outer automorphism of $F_{N}$. Let $T_{\Phi}$ and $T_{\Phi^{-1}}$ be its attracting and repelling trees. Replacing $\Phi$ by a suitable power, we have

$$
2 \operatorname{ind}(\Phi)=\operatorname{ind}_{\text {geo }}\left(T_{\Phi}\right)=\operatorname{ind}_{2}\left(T_{\Phi^{-1}}\right) .
$$

We prove this proposition in Sections $4 \mathrm{~B}$ and $4 \mathrm{C}$.

To study limit trees of iwip automorphisms, we need to state that they have the strongest mixing dynamical property, which is called indecomposability. 
Theorem 2.1. Let $\Phi \in \operatorname{Out}\left(F_{N}\right)$ be an iwip outer automorphism. The attracting tree $T_{\Phi}$ of $\Phi$ is indecomposable.

The proof of this theorem is quite independent of the rest of the paper and is the purpose of Section 2. The proof relies on a key property of iwip automorphisms: they can be represented by (absolute) train-track maps.

\section{Indecomposability of the attracting tree of an iwip automorphism}

Following [Guirardel 2008], a (projective class of) $\mathbb{R}$-tree $T \in \overline{\mathrm{CV}}_{N}$ is indecomposable if for all nondegenerate arcs $I$ and $J$ in $T$, there exists finitely many elements $u_{1}, \ldots, u_{n}$ in $F_{N}$ such that

$$
J \subseteq \bigcup_{i=1}^{n} u_{i} I
$$

and

$$
\forall i=1, \ldots, n-1, \quad u_{i} I \cap u_{i+1} I \text { is a nondegenerate arc. }
$$

The main purpose of this section is to prove this result:

Theorem 2.1. Let $\Phi \in \operatorname{Out}\left(F_{N}\right)$ be an iwip outer automorphism. The attracting tree $T_{\Phi}$ of $\Phi$ is indecomposable.

Before proving this theorem in Section 2C, we collect the results we need from [Bestvina and Handel 1992] and [Gaboriau et al. 1998].

2A. Train-track representative of $\boldsymbol{\Phi}$. The rose $R_{N}$ is the graph with one vertex $*$ and $N$ edges. Its fundamental group $\pi_{1}\left(R_{N}, *\right)$ is naturally identified with the free group $F_{N}$. A marked graph is a finite graph $G$ with a homotopy equivalence $\tau: R_{N} \rightarrow G$. The marking $\tau$ induces an isomorphism

$$
\tau_{*}: F_{N}=\pi_{1}\left(R_{N}, *\right) \stackrel{\cong}{\rightarrow} \pi_{1}\left(G, v_{0}\right),
$$

where $v_{0}=\tau(*)$.

A homotopy equivalence $f: G \rightarrow G$ defines an outer automorphism of $F_{N}$. Indeed, if a path $m$ from $v_{0}$ to $f\left(v_{0}\right)$ is given, $a \mapsto m f(a) m^{-1}$ induces an automorphism $\varphi$ of $\pi_{1}\left(G, v_{0}\right)$, and thus of $F_{N}$ through the marking. Another path $m^{\prime}$ from $v_{0}$ to $f\left(v_{0}\right)$ gives rise to another automorphism $\varphi^{\prime}$ of $F_{N}$ in the same outer class $\Phi$.

A topological representative of $\Phi \in \operatorname{Out}\left(F_{N}\right)$ is an homotopy equivalence $f$ : $G \rightarrow G$ of a marked graph $G$, such that

(i) $f$ maps vertices to vertices,

(ii) $f$ is locally injective on any edge, and

(iii) $f$ induces $\Phi$ on $F_{N} \cong \pi_{1}\left(G, v_{0}\right)$. 
Let $e_{1}, \ldots, e_{p}$ be the edges of $G$ (an orientation is arbitrarily given on each edge, and $e^{-1}$ denotes the edge $e$ with the reverse orientation). The transition matrix of the map $f$ is the $p \times p$ nonnegative matrix $M$ with $(i, j)$-entry equal to the number of times the edge $e_{i}$ occurs in $f\left(e_{j}\right)$ (we say that a path (or an edge) $w$ of a graph $G$ occurs in a path $u$ of $G$ if it is $w$ or its inverse $w^{-1}$ is a subpath of $u$ ).

A topological representative $f: G \rightarrow G$ of $\Phi$ is a train-track map if, moreover, (iv) for all $k \in \mathbb{N}$, the restriction of $f^{k}$ on any edge of $G$ is locally injective, and

(v) any vertex of $G$ has valence at least 3 .

According to [Bestvina and Handel 1992, Theorem 1.7], an iwip outer automorphism $\Phi$ can be represented by a train-track map, with a primitive transition matrix $M$ (i.e., there exists $k \in \mathbb{N}$ such all the entries of $M^{k}$ are strictly positive). Thus the Perron-Frobenius theorem applies. In particular, $M$ has a real dominant eigenvalue $\lambda>1$ associated to a strictly positive eigenvector $u=\left(u_{1}, \ldots, u_{p}\right)$. Indeed, $\lambda$ is the expansion factor of $\Phi: \lambda=\lambda_{\Phi}$. We turn the graph $G$ to a metric space by assigning the length $u_{i}$ to the edge $e_{i}$ (for $i=1, \ldots, p$ ). Since, with respect to this metric, the length of $f\left(e_{i}\right)$ is $\lambda$ times the length of $e_{i}$, we can assume that, on each edge, $f$ is linear of ratio $\lambda$.

We define the set $\mathscr{L}_{2}(f)$ of paths $w$ of combinatorial length 2 (i.e., $w=e e^{\prime}$, where $e, e^{\prime}$ are edges of $\left.G, e^{-1} \neq e^{\prime}\right)$ which occurs in some $f^{k}\left(e_{i}\right)$ for some $k \in \mathbb{N}$ and some edge $e_{i}$ of $G$ :

$\mathscr{L}_{2}(f)=\left\{e e^{\prime}: \exists e_{i}\right.$ edge of $G, \exists k \in \mathbb{N}$ such that $e e^{\prime}$ is a subpath of $\left.f^{k}\left(e_{i}^{ \pm 1}\right)\right\}$.

Since the transition matrix $M$ is primitive, there exists $k \in \mathbb{N}$ such that for any edge $e$ of $G$, for any $w \in \mathscr{L}_{2}(f), w$ occurs in $f^{k}(e)$.

Let $v$ be a vertex of $G$. The Whitehead graph $\mathcal{W}_{v}$ of $v$ is the unoriented graph defined as follows:

- The vertices of $\mathcal{W}_{v}$ are the edges of $G$ with $v$ as terminal vertex.

- There is an edge in $\mathscr{W}_{v}$ between $e$ and $e^{\prime}$ if $e^{\prime} e^{-1} \in \mathscr{L}_{2}(f)$.

As remarked in [Bestvina et al. 1997, Section 2], if $f: G \rightarrow G$ is a train-track representative of an iwip outer automorphism $\Phi$, any vertex of $G$ has a connected Whitehead graph. We summarize the previous discussion:

Proposition 2.2. Let $\Phi \in \operatorname{Out}\left(F_{N}\right)$ be an iwip outer automorphism. There exists a train-track representative $f: G \rightarrow G$ of $\Phi$, with primitive transition matrix $M$ and connected Whitehead graphs of vertices. The edge $e_{i}$ of $G$ is isometric to the segment $\left[0, u_{i}\right]$, where $u=\left(u_{1}, \ldots, u_{p}\right)$ is a Perron-Frobenius eigenvector of $M$. The map $f$ is linear of ratio $\lambda$ on each edge $e_{i}$ of $G$.

Remark 2.3. Let $f: G \rightarrow G$ be a train-track map, with primitive transition matrix $M$ and connected Whitehead graphs of vertices. Then for any path $w=a b$ in $G$ of 
combinatorial length 2 , there exist $w_{1}=a_{1} b_{1}, \ldots, w_{q}=a_{q} b_{q} \in \mathscr{L}_{2}(f)\left(a, b, a_{i}, b_{i}\right.$ edges of $G$ ) such that

- $a_{i+1}=b_{i}^{-1}, i \in\{1, \ldots, q-1\}$, and

- $a=a_{1}$ and $b=b_{q}$.

2B. Construction of $\boldsymbol{T}_{\boldsymbol{\Phi}}$. Let $\Phi \in \operatorname{Out}\left(F_{N}\right)$ be an iwip automorphism, and let $T_{\Phi}$ be its attracting tree. Following [Gaboriau et al. 1998], we recall a concrete construction of the tree $T_{\Phi}$.

We start with a train-track representative $f: G \rightarrow G$ of $\Phi$ as in Proposition 2.2. The universal cover $\tilde{G}$ of $G$ is a simplicial tree, equipped with a distance $d_{0}$ obtained by lifting the distance on $G$. The fundamental group $F_{N}$ acts by deck transformations, and thus by isometries, on $\tilde{G}$. Let $\tilde{f}$ be a lift of $f$ to $\tilde{G}$. This lift $\tilde{f}$ is associated to a unique automorphism $\varphi$ in the outer class $\Phi$, characterized by

$$
\forall u \in F_{N}, \forall x \in \tilde{G}, \quad \varphi(u) \tilde{f}(x)=\tilde{f}(u x) .
$$

For $x, y \in \tilde{G}$ and $k \in \mathbb{N}$, we define:

$$
d_{k}(x, y)=\frac{d_{0}\left(\tilde{f}^{k}(x), \tilde{f}^{k}(y)\right)}{\lambda^{k}} .
$$

The sequence of distances $d_{k}$ is decreasing and converges to a pseudo-distance $d_{\infty}$ on $\tilde{G}$. Identifying points $x, y$ in $\tilde{G}$ which have distance $d_{\infty}(x, y)$ equal to 0 , we obtain the tree $T_{\Phi}$. The free group $F_{N}$ still acts by isometries on $T_{\Phi}$. The quotient map $p: \tilde{G} \rightarrow T_{\Phi}$ is $F_{N}$-equivariant and 1-Lipschitz. Moreover, for any edge $e$ of $\tilde{G}$, for any $k \in \mathbb{N}$, the restriction of $p$ to $f^{k}(e)$ is an isometry. Through $p$ the map $\tilde{f}$ factors to a homothety $H$ of $T_{\Phi}$, of ratio $\lambda_{\Phi}$ :

$$
\forall x \in \tilde{G}, \quad H(p(x))=p(\tilde{f}(x)) .
$$

Property (2-3) leads to

$$
\forall u \in F_{N}, \forall x \in T_{\Phi}, \quad \varphi(u) H(x)=H(u x) .
$$

2C. Indecomposability of $\boldsymbol{T}_{\boldsymbol{\Phi}}$. We say that a path (or an edge) $w$ of the graph $G$ occurs in a path $u$ of the universal cover $\tilde{G}$ of $G$ if $w$ has a lift $\tilde{w}$ that occurs in $u$.

Lemma 2.4. Let $I$ be a nondegenerate arc in $T_{\Phi}$. There exists an arc $I^{\prime}$ in $\tilde{G}$ and an integer $k$ such that

- $p\left(I^{\prime}\right) \subseteq I$, and

- any element of $\mathscr{L}_{2}(f)$ occurs in $H^{k}\left(I^{\prime}\right)$. 
Proof. Let $I \subset T_{\Phi}$ be a nondegenerate arc. There exists an edge $e$ of $\tilde{G}$ such that $I_{0}=p(e) \cap I$ is a nondegenerate $\operatorname{arc:} I_{0}=[x, y]$. We choose $k_{1} \in \mathbb{N}$ such that $d_{\infty}\left(H^{k_{1}}(x), H^{k_{1}}(y)\right)>L$ where

$$
L=2 \max \left\{u_{i}=\left|e_{i}\right| \mid e_{i} \text { edge of } G\right\} .
$$

Let $x^{\prime}, y^{\prime}$ be the points in $e$ such that $p\left(x^{\prime}\right)=x, p\left(y^{\prime}\right)=y$, and let $I^{\prime}$ be the arc $\left[x^{\prime}, y^{\prime}\right]$. Since $p$ maps $f^{k_{1}}(e)$ isometrically into $T_{\Phi}$, we obtain that

$$
d_{0}\left(f^{k_{1}}\left(x^{\prime}\right), f^{k_{1}}\left(y^{\prime}\right)\right) \geq L .
$$

Hence there exists an edge $e^{\prime}$ of $\tilde{G}$ contained in $\left[f^{k_{1}}\left(x^{\prime}\right), f^{k_{1}}\left(y^{\prime}\right)\right]$. Moreover, for any $k_{2} \in \mathbb{N}$, the path $f^{k_{2}}\left(e^{\prime}\right)$ isometrically injects in $\left[H^{k_{1}+k_{2}}(x), H^{k_{1}+k_{2}}(y)\right]$. We take $k_{2}$ big enough so that any path in $\mathscr{L}_{2}(f)$ occurs in $f^{k_{2}}\left(e^{\prime}\right)$. Then $k=k_{1}+k_{2}$ is suitable.

Proof of Theorem 2.1. Let $I, J$ be two nontrivial arcs in $T_{\Phi}$. We have to prove that $I$ and $J$ satisfy properties (2-1) and (2-2). Since $H$ is a homeomorphism, and because of (2-4), we can replace $I$ and $J$ by $H^{k}(I)$ and $H^{k}(J)$, accordingly, for some $k \in \mathbb{N}$.

We consider an $\operatorname{arc} I^{\prime}$ in $\tilde{G}$ and an integer $k \in \mathbb{N}$ as given by Lemma 2.4. Let $x, y$ be the endpoints of the arc $H^{k}(J): H^{k}(J)=[x, y]$. Let $x^{\prime}, y^{\prime}$ be points in $\tilde{G}$ such that $p\left(x^{\prime}\right)=x, p\left(y^{\prime}\right)=y$, and let $J^{\prime}$ be the arc $\left[x^{\prime}, y^{\prime}\right]$. According to Remark 2.3, there exist $w_{1}, \ldots, w_{n}$ such that

- $w_{i}$ is a lift of some path in $\mathscr{L}_{2}(f)$,

- $J^{\prime} \subseteq \bigcup_{i=1}^{n} w_{i}$, and

- $w_{i} \cap w_{i+1}$ is an edge.

Since Lemma 2.4 ensures that any element of $\mathscr{L}_{2}(f)$ occurs in $H^{k}\left(I^{\prime}\right)$, we deduce that $H^{k}(I)$ and $H^{k}(J)$ satisfy properties (2-1) and (2-2).

\section{Index of an outer automorphism}

An automorphism $\varphi$ of the free group $F_{N}$ extends to a homeomorphism $\partial \varphi$ of the boundary at infinity $\partial F_{N}$. We denote by $\operatorname{Fix}(\varphi)$ the fixed subgroup of $\varphi$. It is a finitely generated subgroup of $F_{N}$ and thus its boundary $\partial \mathrm{Fix}(\varphi)$ naturally embeds in $\partial F_{N}$. Elements of $\partial \operatorname{Fix}(\varphi)$ are fixed by $\partial \varphi$ and they are called singular. Nonsingular fixed points of $\partial \varphi$ are called regular. A fixed point $X$ of $\partial \varphi$ is attracting (resp. repelling) if it is regular and if there exists an element $u$ in $F_{N}$ such that $\varphi^{n}(u)$ (resp. $\varphi^{-n}(u)$ ) converges to $X$. The set of fixed points of $\partial \varphi$ is denoted by $\operatorname{Fix}(\partial \varphi)$.

Following Nielsen, fixed points of $\partial \varphi$ have been classified by Gaboriau, Jaeger, Levitt and, Lustig: 
Proposition 3.1 [Gaboriau et al. 1998, Proposition 1.1]. Let $\varphi$ be an automorphism of the free group $F_{N}$, and $X$ a fixed point of $\partial \varphi$. Exactly one of the following occurs:

(1) $X$ is in the boundary of the fixed subgroup of $\varphi$.

(2) $X$ is attracting.

(3) $X$ is repelling.

We denote by $\operatorname{Att}(\varphi)$ the set of attracting fixed points of $\partial \varphi$. The fixed subgroup Fix $(\varphi)$ acts on the set $\operatorname{Att}(\varphi)$ of attracting fixed points.

In [Gaboriau et al. 1998] the following index of the automorphism $\varphi$ is defined:

$$
\operatorname{ind}(\varphi)=\frac{1}{2} \#(\operatorname{Att}(\varphi) / \operatorname{Fix}(\varphi))+\operatorname{rank}(\operatorname{Fix}(\varphi))-1
$$

If $\varphi$ has a trivial fixed subgroup, the above definition is simpler:

$$
\operatorname{ind}(\varphi)=\frac{1}{2} \# \operatorname{Att}(\varphi)-1 .
$$

Let $u$ be an element of $F_{N}$ and let $i_{u}$ be the corresponding inner automorphism of $F_{N}$ :

$$
\forall w \in F_{N}, \quad i_{u}(w)=u w u^{-1} .
$$

The inner automorphism $i_{u}$ extends to the boundary of $F_{N}$ as left multiplication by $u$ :

$$
\forall X \in \partial F_{N}, \quad \partial i_{u}(X)=u X .
$$

The group $\operatorname{Inn}\left(F_{N}\right)$ of inner automorphisms of $F_{N}$ acts by conjugacy on the automorphisms in an outer class $\Phi$. Following Nielsen, two automorphisms, $\varphi, \varphi^{\prime} \in \Phi$ are isogredient if they are conjugated by some inner automorphism $i_{u}$ :

$$
\varphi^{\prime}=i_{u} \circ \varphi \circ i_{u^{-1}}=i_{u \varphi(u)^{-1}} \circ \varphi .
$$

In this case, the actions of $\partial \varphi$ and $\partial \varphi^{\prime}$ on $\partial F_{N}$ are conjugate by the left multiplication by $u$. In particular, a fixed point $X^{\prime}$ of $\partial \varphi^{\prime}$ is a translate $X^{\prime}=u X$ of a fixed point $X$ of $\partial \varphi$. Two isogredient automorphisms have the same index: this is the index of the isogrediency class. An isogrediency class $[\varphi]$ is essential if it has positive index: ind $([\varphi])>0$. We note that essential isogrediency classes are principal in the sense of [Feighn and Handel 2011], but the converse is not true.

The index of the outer automorphism $\Phi$ is the sum, over all essential isogrediency classes of automorphisms $\varphi$ in the outer class $\Phi$, of their indices, or alternatively:

$$
\operatorname{ind}(\Phi)=\sum_{[\varphi] \in \Phi / \operatorname{Inn}\left(F_{N}\right)} \max (0 ; \operatorname{ind}(\varphi)) .
$$

We adapt the notion of forward rotationless outer automorphism of [Feighn and Handel 2011] to our purpose. We denote by $\operatorname{Per}(\varphi)$ the set of elements of $F_{N}$ fixed 
by some positive power of $\varphi$ :

$$
\operatorname{Per}(\varphi)=\bigcup_{n \in \mathbb{N}^{*}} \operatorname{Fix}\left(\varphi^{n}\right)
$$

and by $\operatorname{Per}(\partial \varphi)$ the set of elements of $\partial F_{N}$ fixed by some positive power of $\partial \varphi$ :

$$
\operatorname{Per}(\partial \varphi)=\bigcup_{n \in \mathbb{N}^{*}} \operatorname{Fix}\left(\partial \varphi^{n}\right) .
$$

Definition 3.2. An outer automorphism $\Phi \in \operatorname{Out}\left(F_{N}\right)$ is FR if:

(FR1) for any automorphism $\varphi \in \Phi, \operatorname{Per}(\varphi)=\operatorname{Fix}(\varphi)$ and $\operatorname{Per}(\partial \varphi)=\operatorname{Fix}(\partial \varphi)$, and (FR2) if $\psi$ is an automorphism in the outer class $\Phi^{n}$ for some $n>0$, with ind $(\psi)$ positive, then there exists an automorphism $\varphi$ in $\Phi$ such that $\psi=\varphi^{n}$.

Proposition 3.3. Let $\Phi \in \operatorname{Out}\left(F_{N}\right)$. There exists $k \in \mathbb{N}^{*}$ such that $\Phi^{k}$ is $F R$.

Proof. By [Levitt and Lustig 2000, Theorem 1] there exists a power $\Phi^{k}$ with (FR1). An automorphism $\varphi \in \operatorname{Aut}\left(F_{N}\right)$ with positive index $\operatorname{ind}(\varphi)>0$ is principal in the sense of [Feighn and Handel 2011, Definition 3.1]. Thus our property (FR2) is a consequence of the forward rotationless property of [loc. cit., Definition 3.13]. By [loc. cit., Lemma 4.43] there exists a power $\Phi^{k \ell}$ which is forward rotationless and thus which satisfies (FR2).

\section{Indices}

4A. Botany of trees. We recall in this section the classification of trees in the boundary of outer space, given in [Coulbois and Hilion 2010].

Gaboriau and Levitt [1995] introduced an index for a tree $T$ in $\overline{\mathrm{CV}}_{N}$, we call it the geometric index and denote it by $\operatorname{ind}_{\mathrm{geo}}(T)$. It is defined using the valence of the branch points, of the $\mathbb{R}$-tree $T$, with an action of the free group by isometries:

$$
\operatorname{ind}_{\mathrm{geo}}(T)=\sum_{[P] \in T / F_{N}} \operatorname{ind}_{\mathrm{geo}}(P)
$$

where the local index of a point $P$ in $T$ is

$$
\operatorname{ind}_{\mathrm{geo}}(P)=\#\left(\pi_{0}(T \backslash\{P\}) / \operatorname{Stab}(P)\right)+2 \operatorname{rank}(\operatorname{Stab}(P))-2 .
$$

Gaboriau and Levitt proved that the geometric index of a geometric tree is equal to $2 N-2$ and that for any tree in the compactification of outer space $\overline{\mathrm{CV}}_{N}$ the geometric index is bounded above by $2 N-2$. Moreover, they proved that the trees in $\overline{\mathrm{CV}}_{N}$ with geometric index equal to $2 N-2$ are precisely the geometric trees.

If, moreover, $T$ has dense orbits, Levitt and Lustig [2003; 2008] defined the map $2: \partial F_{N} \rightarrow \hat{T}$, characterized as follows: 
Proposition 4.1. Let $T$ be an $\mathbb{R}$-tree in $\overline{\mathrm{CV}}_{N}$ with dense orbits. There exists a unique map $2: \partial F_{N} \rightarrow \hat{T}$ such that for any sequence $\left(u_{n}\right)_{n \in \mathbb{N}}$ of elements of $F_{N}$ which converges to $X \in \partial F_{N}$, and any point $P \in T$, if the sequence of points $\left(u_{n} P\right)_{n \in \mathbb{N}}$ converges to a point $Q \in \hat{T}$, then $\mathscr{Q}(X)=Q$. Moreover, 2 is onto.

Let us consider the case of a tree $T$ dual to a measured foliation $(\mathscr{F}, \mu)$ on a hyperbolic surface $S$ with boundary ( $T$ is a surface tree). Let $\tilde{F}_{\text {f }}$ be the lift of $\mathscr{F}$ to the universal cover $\tilde{S}$ of $S$. The boundary at infinity of $\tilde{S}$ is homeomorphic to $\partial F_{N}$. On the one hand, a leaf $\ell$ of $\tilde{\mathscr{F}}$ defines a point in $T$. On the other hand, the ends of $\ell$ define points in $\partial F_{N}$. The map 2 precisely sends the ends of $\ell$ to the point in $T$. The Poincaré-Lefschetz index of the foliation $\mathscr{F}$ can be computed from the cardinal of the fibers of the map 2. This leads to the following definition of the 2-index of an $\mathbb{R}$-tree $T$ in a more general context.

Let $T$ be an $\mathbb{R}$-tree in $\overline{\mathrm{CV}}_{N}$ with dense orbits. The 2-index of the tree $T$ is defined by

$$
\operatorname{ind}_{2}(T)=\sum_{[P] \in \hat{T} / F_{N}} \max \left(0 ; \operatorname{ind}_{2}(P)\right),
$$

where the local index of a point $P$ in $T$ is

$$
\operatorname{ind}_{2}(P)=\#\left(\mathscr{Q}_{r}^{-1}(P) / \operatorname{Stab}(P)\right)+2 \operatorname{rank}(\operatorname{Stab}(P))-2
$$

with $\mathscr{2}_{r}^{-1}(P)=\mathscr{2}^{-1}(P) \backslash \partial \operatorname{Stab}(P)$ the regular fiber of $P$.

Levitt and Lustig [2003] proved that points in $\partial T$ have exactly one pre-image by 2 . Thus, only points in $\bar{T}$ contribute to the 2 -index of $T$.

We proved in [Coulbois and Hilion 2010] that the 2-index of an $\mathbb{R}$-tree in the boundary of outer space with dense orbits is bounded above by $2 N-2$. And it is equal to $2 N-2$ if and only if it is of surface type.

The botanical classification in [Coulbois and Hilion 2010] of a tree $T$ with a

\begin{tabular}{|c|c|c|}
\hline & $\begin{array}{c}\text { geometric } \\
\operatorname{ind}_{\mathrm{geo}}(T)=2 N-2\end{array}$ & $\begin{array}{c}\text { not geometric } \\
\operatorname{ind}_{\text {geo }}(T)<2 N-2\end{array}$ \\
\hline Surface type: $\operatorname{ind}_{2}(T)=2 N-2$ & surface & pseudo-surface \\
\hline Levitt type: $\operatorname{ind}_{2}(T)<2 N-2$ & Levitt & pseudo-Levitt \\
\hline
\end{tabular}
minimal very small indecomposable action of $F_{N}$ by isometries is as follows:

The following remark is not necessary for the sequel of the paper, but may help the reader's intuition.

Remark. In [Coulbois et al. 2008a; 2008b], in collaboration with Lustig, we defined and studied the dual lamination of an $\mathbb{R}$-tree $T$ with dense orbits:

$$
L(T)=\left\{(X, Y) \in \partial^{2} F_{N} \mid \mathscr{2}(X)=2(Y)\right\} .
$$


The 2-index of $T$ can be interpreted as the index of this dual lamination.

Using the dual lamination, with Lustig [Coulbois et al. 2009], we defined the compact heart $K_{A} \subseteq \bar{T}$ (for a basis $A$ of $F_{N}$ ). We proved that the tree $T$ is completely encoded by a system of partial isometries $S_{A}=\left(K_{A}, A\right)$. We also proved that the tree $T$ is geometric if and only if the compact heart $K_{A}$ is a finite tree (that is to say the convex hull of finitely many points). In [Coulbois and Hilion 2010] we used the Rips machine on the system of isometries $S_{A}$ to get the bound on the 2-index of $T$. In particular, an indecomposable tree $T$ is of Levitt type if and only if the Rips machine never halts.

4B. Geometric index. As in Section 2B, an iwip outer automorphism $\Phi$ has an expansion factor $\lambda_{\Phi}>1$, an attracting $\mathbb{R}$-tree $T_{\Phi}$ in $\partial \mathrm{CV}_{N}$. For each automorphism $\varphi$ in the outer class $\Phi$ there is a homothety $H$ of the metric completion $\bar{T}_{\Phi}$, of ratio $\lambda_{\Phi}$, such that

$$
\forall P \in \bar{T}_{\Phi}, \forall u \in F_{N}, \quad H(u P)=\varphi(u) H(P) .
$$

In addition, the action of $\Phi$ on the compactification of Culler and Vogtmann's outer space has north-south dynamics and the projective class of $T_{\Phi}$ is the attracting fixed point [Levitt and Lustig 2003]. Of course the attracting trees of $\Phi$ and $\Phi^{n}$ $(n>0)$ are equal.

For the attracting tree $T_{\Phi}$ of the iwip outer automorphism $\Phi$, the geometric index is well understood.

Proposition 4.2 [Gaboriau et al. 1998, Section 4]. Let $\Psi$ be an iwip outer automorphism. There exists a power $\Phi=\Psi^{k}(k>0)$ of $\Psi$ such that

$$
2 \operatorname{ind}(\Phi)=\operatorname{ind}_{\mathrm{geo}}\left(T_{\Phi}\right)
$$

where $T_{\Phi}$ is the attracting tree of $\Phi$ (and of $\Psi$ ).

4C. 2-index. Let $\Phi$ be an iwip outer automorphism of $F_{N}$. Let $T_{\Phi}$ be its attracting tree. The action of $F_{N}$ on $T_{\Phi}$ has dense orbits.

Let $\varphi$ an automorphism in the outer class $\Phi$. The homothety $H$ associated to $\varphi$ extends continuously to an homeomorphism of the boundary at infinity of $T_{\Phi}$ which we still denote by $H$. We get from Proposition 4.1 and identity (4-1):

$$
\forall X \in \partial F_{N}, \quad 2(\partial \varphi(X))=H(2(X)) .
$$

We are going to prove that the 2-index of $T_{\Phi}$ is twice the index of $\Phi^{-1}$. As mentioned in the introduction for geometric automorphisms both these numbers are equal to $2 N-2$ and thus we restrict to the study of nongeometric automorphisms. For the rest of this section we assume that $\Phi$ is nongeometric. This will be used in two ways: 
- The action of $F_{N}$ on $T_{\Phi}$ is free.

- For any $\varphi$ in the outer class $\Phi$, all the fixed points of $\varphi$ in $\partial F_{N}$ are regular.

Let $C_{H}$ be the center of the homothety $H$. The following Lemma is essentially contained in [Gaboriau et al. 1998], although the map 2 is not used there.

Lemma 4.3. Let $\Phi \in \operatorname{Out}\left(F_{N}\right)$ be a FR nongeometric iwip outer automorphism. Let $T_{\Phi}$ be the attracting tree of $\Phi$. Let $\varphi \in \Phi$ be an automorphism in the outer class $\Phi$, and let $H$ be the homothety of $T_{\Phi}$ associated to $\varphi$, with $C_{H}$ its center. The 2-fiber of $C_{H}$ is the set of repelling points of $\varphi$.

Proof. Let $X \in \partial F_{N}$ be a repelling point of $\partial \varphi$. By definition there exists an element $u \in F_{N}$ such that the sequence $\left(\varphi^{-n}(u)\right)_{n}$ converges towards $X$. By (4-1),

$$
\varphi^{-n}(u) C_{H}=\varphi^{-n}(u) H^{-n}\left(C_{H}\right)=H^{-n}\left(u C_{H}\right) .
$$

The homothety $H^{-1}$ is strictly contracting and therefore the sequence of points $\left(\varphi^{-n}(u) C_{H}\right)_{n}$ converges towards $C_{H}$. By Proposition 4.1 we get that $2(X)=C_{H}$.

Conversely let $X \in 2^{-1}\left(C_{H}\right)$ be a point in the 2-fiber of $C_{H}$. Using the identity (4-2), $\partial \varphi(X)$ is also in the 2-fiber. The 2-fiber is finite by [Coulbois and Hilion 2010, Corollary 5.4], $X$ is a periodic point of $\partial \varphi$. Since $\Phi$ satisfies property (FR1), $X$ is a fixed point of $\partial \varphi$. From [Gaboriau et al. 1998, Lemma 3.5], attracting fixed points of $\partial \varphi$ are mapped by 2 to points in the boundary at infinity $\partial T_{\Phi}$. Thus $X$ has to be a repelling fixed point of $\partial \varphi$.

Proposition 4.4. Let $\Phi \in \operatorname{Out}\left(F_{N}\right)$ be a FR nongeometric iwip outer automorphism. Let $T_{\Phi}$ be the attracting tree of $\Phi$. Then

$$
2 \operatorname{ind}\left(\Phi^{-1}\right)=\operatorname{ind}_{2}\left(T_{\Phi}\right) .
$$

Proof. To each automorphism $\varphi$ in the outer class $\Phi$ is associated a homothety $H$ of $T_{\Phi}$ and the center $C_{H}$ of this homothety. As the action of $F_{N}$ on $T_{\Phi}$ is free, two automorphisms are isogredient if and only if the corresponding centers are in the same $F_{N}$-orbit.

The index of $\Phi^{-1}$ is the sum over all essential isogrediency classes of automorphism $\varphi^{-1}$ in $\Phi^{-1}$ of the index of $\varphi^{-1}$. For each of these automorphisms the index 2 ind $\left(\varphi^{-1}\right)$ is equal by Lemma 4.3 to the contribution $\# 2^{-1}\left(C_{H}\right)$ of the orbit of $C_{H}$ to the 2 index of $T_{\Phi}$.

Conversely, let now $P$ be a point in $\bar{T}_{\Phi}$ with at least three elements in its 2 -fiber. Let $\varphi$ be an automorphism in $\Phi$ and let $H$ be the homothety of $T_{\Phi}$ associated to $\varphi$. For any integer $n$, the 2 -fiber $2^{-1}\left(H^{n}(P)\right)=\partial \varphi^{n}\left(\mathscr{Q}^{-1}(P)\right)$ of $H^{n}(P)$ also has at least three elements. By [Coulbois and Hilion 2010, Theorem 5.3] there are finitely many orbits of such points in $T_{\Phi}$ and thus we can assume that $H^{n}(P)=w P$ for some $w \in F_{N}$ and some integer $n>0$. Then $P$ is the center of the homothety 
$w^{-1} H^{n}$ associated to $i_{w^{-1}} \circ \varphi^{n}$. Since $\Phi$ satisfies property (FR2), $P$ is the center of a homothety $u H$ associated to $i_{u} \circ \varphi$ for some $u \in F_{N}$. This concludes the proof of the equality of the indices.

This proposition can alternatively be deduced from the techniques of [Handel and Mosher 2011].

\section{Botanical classification of irreducible automorphisms}

Theorem 5.1. Let $\Phi$ be an iwip outer automorphism of $F_{N}$. Let $T_{\Phi}$ and $T_{\Phi^{-1}}$ be its attracting and repelling trees. Then, the 2-index of the attracting tree is equal to the geometric index of the repelling tree:

$$
\operatorname{ind}_{2}\left(T_{\Phi}\right)=\operatorname{ind}_{\text {geo }}\left(T_{\Phi^{-1}}\right) .
$$

Proof. First, if $\Phi$ is geometric, then the trees $T_{\Phi}$ and $T_{\Phi^{-1}}$ have maximal geometric indices $2 N-2$. On the other hand the trees $T_{\Phi}$ and $T_{\Phi^{-1}}$ are surface trees and thus their 2-indices are also maximal:

$$
\operatorname{ind}_{\mathrm{geo}}\left(T_{\Phi}\right)=\operatorname{ind}_{2}\left(T_{\Phi}\right)=\operatorname{ind}_{\mathrm{geo}}\left(T_{\Phi^{-1}}\right)=\operatorname{ind}_{2}\left(T_{\Phi^{-1}}\right)=2 N-2 .
$$

We now assume that $\Phi$ is not geometric and we can apply Propositions 4.2 and 4.4 to get the desired equality.

From Theorem 5.1 and from the characterization of geometric and surface-type trees by the maximality of the indices we get

Theorem 5.2. Let $\Phi$ be an iwip outer automorphism of $F_{N}$. Let $T_{\Phi}$ and $T_{\Phi^{-1}}$ be its attracting and repelling trees. Then exactly one of the following occurs:

(1) $T_{\Phi}$ and $T_{\Phi^{-1}}$ are surface trees.

(2) $T_{\Phi}$ is Levitt and $T_{\Phi^{-1}}$ is pseudo-surface.

(3) $T_{\Phi^{-1}}$ is Levitt and $T_{\Phi}$ is pseudo-surface.

(4) $T_{\Phi}$ and $T_{\Phi^{-1}}$ are pseudo-Levitt.

Proof. The trees $T_{\Phi}$ and $T_{\Phi^{-1}}$ are indecomposable by Theorem 2.1 and thus they are either of surface type or of Levitt type by [Coulbois and Hilion 2010, Proposition 5.14]. Recall, from [Gaboriau and Levitt 1995] (see also [Coulbois and Hilion 2010, Theorem 5.9] or [Coulbois et al. 2009, Corollary 6.1]) that $T_{\Phi}$ is geometric if and only if its geometric index is maximal:

$$
\operatorname{ind}_{\mathrm{geo}}\left(T_{\Phi}\right)=2 N-2 .
$$

From [Coulbois and Hilion 2010, Theorem 5.10], $T_{\Phi}$ is of surface type if and only if its 2 -index is maximal:

$$
\operatorname{ind}_{2}\left(T_{\Phi}\right)=2 N-2
$$


The theorem now follows from Theorem 5.1.

Let $\Phi \in \operatorname{Out}\left(F_{N}\right)$ be an iwip outer automorphism.

The outer automorphism $\Phi$ is geometric if both its attracting and repelling trees $T_{\Phi}$ and $T_{\Phi^{-1}}$ are geometric. This is equivalent to saying that $\Phi$ is induced by a pseudo-Anosov homeomorphism of a surface with boundary; see [Guirardel 2005] and [Handel and Mosher 2007]. This is case (1) of Theorem 5.2.

The outer automorphism $\Phi$ is parageometric if its attracting tree $T_{\Phi}$ is geometric but its repelling tree $T_{\Phi^{-1}}$ is not. This is case (2) of Theorem 5.2.

The outer automorphism $\Phi$ is pseudo-Levitt if both its attracting and repelling trees are not geometric. This is case (4) of Theorem 5.2

We now bring expansion factors into play. An iwip outer automorphism $\Phi$ of $F_{N}$ has an expansion factor $\lambda_{\Phi}>1$ : it is the exponential growth rate of (nonfixed) conjugacy classes under iteration of $\Phi$.

If $\Phi$ is geometric, the expansion factor of $\Phi$ is equal to the expansion factor of the associated pseudo-Anosov mapping class and thus $\lambda_{\Phi}=\lambda_{\Phi^{-1}}$.

Handel and Mosher [2007] proved that if $\Phi$ is a parageometric outer automorphism of $F_{N}$ then $\lambda_{\Phi}>\lambda_{\Phi^{-1}}$ (see also [Behrstock et al. 2010]). Examples are also given by Gautero [2007].

For pseudo-Levitt outer automorphisms of $F_{N}$ nothing can be said on the comparison of the expansion factors of the automorphism and its inverse. On one hand, Handel and Mosher [2007, Introduction] gave an explicit example of a nongeometric automorphism with $\lambda_{\Phi}=\lambda_{\Phi^{-1}}$ : thus this automorphism is pseudo-Levitt. On the other hand, there are examples of pseudo-Levitt automorphisms with $\lambda_{\Phi}>\lambda_{\Phi^{-1}}$. Let $\varphi \in \operatorname{Aut}\left(F_{3}\right)$ be the automorphism such that

$$
\begin{aligned}
& \varphi: a \mapsto b \quad \text { and } \quad \varphi^{-1}: a \mapsto c \\
& b \mapsto a c \quad b \mapsto a \\
& c \mapsto a \quad c \mapsto c^{-1} b
\end{aligned}
$$

Let $\Phi$ be its outer class. Then $\Phi^{6}$ is FR, has index ind $\left(\Phi^{6}\right)=\frac{3}{2}<2$. The expansion factor is $\lambda_{\Phi} \simeq 1,3247$. The outer automorphism $\Phi^{-3}$ is FR, has index $\operatorname{ind}\left(\Phi^{-3}\right)=$ $\frac{1}{2}<2$. The expansion factor is $\lambda_{\Phi^{-1}} \simeq 1,4655>\lambda_{\Phi}$. The computation of these two indices can be achieved using the algorithm of [Jullian 2009].

Now that we have classified outer automorphisms of $F_{N}$ into four categories, questions of genericity naturally arise. In particular, is a generic outer automorphism of $F_{N}$ iwip, pseudo-Levitt and with distinct expansion factors? This was suggested in [Handel and Mosher 2007], in particular for statistical genericity: given a set of generators of $\operatorname{Out}\left(F_{N}\right)$ and considering the word metric associated 
to it, is it the case that

$$
\lim _{k \rightarrow \infty} \frac{\left.\#\left(\text { pseudo-Levitt iwip with } \lambda_{\Phi} \neq \lambda_{\Phi^{-1}}\right) \cap B(k)\right)}{\# B(k)}=1,
$$

where $B(k)$ is the ball of radius $k$, centered at 1 , in $\operatorname{Out}\left(F_{N}\right)$ ?

5A. Botanical memo. In this section we give a glossary of our classification of automorphisms for the working mathematician.

For a FR iwip outer automorphism $\Phi$ of $F_{N}$, we used 6 indices which are related in the following way:

$$
\begin{aligned}
2 \operatorname{ind}(\Phi) & =\operatorname{ind}_{\mathrm{geo}}\left(T_{\Phi}\right)=\operatorname{ind}_{2}\left(T_{\Phi^{-1}}\right), \\
2 \operatorname{ind}\left(\Phi^{-1}\right) & =\operatorname{ind}_{\mathrm{geo}}\left(T_{\Phi^{-1}}\right)=\operatorname{ind}_{2}\left(T_{\Phi}\right) .
\end{aligned}
$$

\begin{tabular}{|c|c|c|c|}
\hline Automorphisms & & Trees & Indices \\
\hline $\begin{array}{c}\Phi \text { geometric } \\
\text { 业 } \\
\Phi^{-1} \text { geometric }\end{array}$ & & $\begin{array}{c}T_{\Phi} \text { and } T_{\Phi^{-1}} \text { geometric } \\
\text { 星 } \\
T_{\Phi} \text { surface } \\
\text { 企 } \\
T_{\Phi^{-1}} \text { surface }\end{array}$ & $\Leftrightarrow \operatorname{ind}(\Phi)=\operatorname{ind}\left(\Phi^{-1}\right)=N-1$ \\
\hline$\Phi$ parageometric & $\Leftrightarrow$ & 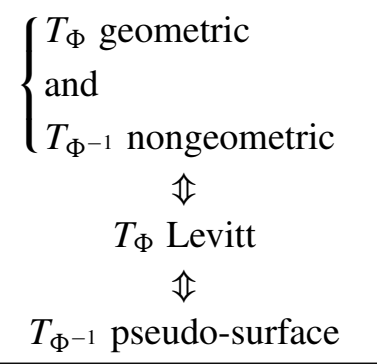 & $\left\{\begin{array}{l}\operatorname{ind}(\Phi)=N-1 \\
\text { and } \\
\operatorname{ind}\left(\Phi^{-1}\right)<N-1\end{array}\right.$ \\
\hline $\begin{array}{c}\Phi \text { pseudo-Levitt } \\
\mathbb{1} \\
\Phi^{-1} \text { pseudo-Levitt }\end{array}$ & $\Leftrightarrow$ & $\begin{array}{c}T_{\Phi}, T_{\Phi^{-1}} \text { nongeometric } \\
\mathbb{1} \\
T_{\Phi} \text { pseudo-Levitt } \\
\text { 氽 } \\
T_{\Phi^{-1}} \text { pseudo-Levitt }\end{array}$ & $\left\{\begin{array}{l}\operatorname{ind}(\Phi)<N-1 \\
\text { and } \\
\operatorname{ind}\left(\Phi^{-1}\right)<N-1\end{array}\right.$ \\
\hline
\end{tabular}

All these indices are bounded above by $2 N-2$. We sum up our Theorem 5.2 in the following table.

\section{Acknowledgements}

We thank Martin Lustig for his constant interest in our work and the referee for the suggested improvements. 


\section{References}

[Behrstock et al. 2010] J. Behrstock, M. Bestvina, and M. Clay, "Growth of intersection numbers for free group automorphisms", J. Topol. 3:2 (2010), 280-310. MR 2011j:20072 Zbl 1209.20031

[Bestvina 2002] M. Bestvina, "R-trees in topology, geometry, and group theory", pp. 55-91 in Handbook of geometric topology, edited by R. J. Daverman and R. B. Sher, North-Holland, Amsterdam, 2002. MR 2003b:20040 Zbl 0998.57003

[Bestvina and Feighn 1992] M. Bestvina and M. Feighn, "A combination theorem for negatively curved groups”, J. Differential Geom. 35:1 (1992), 85-101. MR 93d:53053 Zbl 0724.57029

[Bestvina and Handel 1992] M. Bestvina and M. Handel, "Train tracks and automorphisms of free groups", Ann. of Math. (2) 135:1 (1992), 1-51. MR 92m:20017 Zbl 0757.57004

[Bestvina et al. 1997] M. Bestvina, M. Feighn, and M. Handel, "Laminations, trees, and irreducible automorphisms of free groups", Geom. Funct. Anal. 7:2 (1997), 215-244. MR 98c:20045 Zbl 0884.57002

[Brinkmann 2000] P. Brinkmann, "Hyperbolic automorphisms of free groups", Geom. Funct. Anal. 10:5 (2000), 1071-1089. MR 2001m:20061 Zbl 0970.20018

[Coulbois and Hilion 2010] T. Coulbois and A. Hilion, "Rips induction: index of the dual lamination of an $\mathbb{R}$-tree”, preprint, 2010. arXiv 1002.0972

[Coulbois et al. 2008a] T. Coulbois, A. Hilion, and M. Lustig, " $\mathbb{R}$-trees and laminations for free groups. I. Algebraic laminations”, J. Lond. Math. Soc. (2) 78:3 (2008), 723-736. MR 2010e:20038

[Coulbois et al. 2008b] T. Coulbois, A. Hilion, and M. Lustig, "R-trees and laminations for free groups. II. The dual lamination of an R-tree", J. Lond. Math. Soc. (2) 78:3 (2008), 737-754. MR 2010h:20056 Zbl 1198.20023

[Coulbois et al. 2009] T. Coulbois, A. Hilion, and M. Lustig, " $\mathbb{R}-$ trees, dual laminations and compact systems of partial isometries", Math. Proc. Cambridge Philos. Soc. 147:2 (2009), 345-368. MR 2010m:20031 Zbl 05617513

[Feighn and Handel 2011] M. Feighn and M. Handel, "The recognition theorem for Out $\left(F_{n}\right)$ ", Groups Geom. Dyn. 5:1 (2011), 39-106. MR 2012b:20061 Zbl 05973328

[Gaboriau and Levitt 1995] D. Gaboriau and G. Levitt, "The rank of actions on R-trees", Ann. Sci. École Norm. Sup. (4) 28:5 (1995), 549-570. MR 97c:20039 Zbl 0835.20038

[Gaboriau et al. 1998] D. Gaboriau, A. Jaeger, G. Levitt, and M. Lustig, "An index for counting fixed points of automorphisms of free groups”, Duke Math. J. 93:3 (1998), 425-452. MR 99f:20051 Zbl 0946.20010

[Gautero 2007] F. Gautero, "Combinatorial mapping-torus, branched surfaces and free group automorphisms”, Ann. Sc. Norm. Super. Pisa Cl. Sci. (5) 6:3 (2007), 405-440. MR 2008m:20043 Zbl 1173.20017

[Guirardel 2005] V. Guirardel, "Cœur et nombre d'intersection pour les actions de groupes sur les arbres”, Ann. Sci. École Norm. Sup. (4) 38:6 (2005), 847-888. MR 2007e:20055

[Guirardel 2008] V. Guirardel, "Actions of finitely generated groups on $\mathbb{R}$-trees", Ann. Inst. Fourier (Grenoble) 58:1 (2008), 159-211. MR 2009g:20044 Zbl 1187.20020

[Handel and Mosher 2007] M. Handel and L. Mosher, "Parageometric outer automorphisms of free groups", Trans. Amer. Math. Soc. 359:7 (2007), 3153-3183. MR 2008c:20045 Zbl 1120.20042

[Handel and Mosher 2011] M. Handel and L. Mosher, Axes in outer space, Mem. Amer. Math. Soc. 1004, Amer. Math. Soc., Providence, RI, 2011. MR 2858636 Zbl 05947410 arXiv math/0605355

[Jullian 2009] Y. Jullian, Représentations géométriques des systèmes dynamiques substitutifs par substitutions d'arbre, $\mathrm{PhD}$ thesis, Université Aix-Marseille II, 2009. 
[Levitt 1993] G. Levitt, "La dynamique des pseudogroupes de rotations", Invent. Math. 113:3 (1993), 633-670. MR 94k:58158 Zbl 0791.58055

[Levitt and Lustig 2000] G. Levitt and M. Lustig, "Periodic ends, growth rates, Hölder dynamics for automorphisms of free groups", Comment. Math. Helv. 75:3 (2000), 415-429. MR 2002g:20047 Zbl 0965.20026

[Levitt and Lustig 2003] G. Levitt and M. Lustig, "Irreducible automorphisms of $F_{n}$ have northsouth dynamics on compactified outer space", J. Inst. Math. Jussieu 2:1 (2003), 59-72. MR 2004a: 20046 Zbl 1034.20038

[Levitt and Lustig 2008] G. Levitt and M. Lustig, "Automorphisms of free groups have asymptotically periodic dynamics", J. Reine Angew. Math. 619 (2008), 1-36. MR 2009m:20034 Zbl 1157. 20017

[Rivin 2008] I. Rivin, "Walks on groups, counting reducible matrices, polynomials, and surface and free group automorphisms", Duke Math. J. 142:2 (2008), 353-379. MR 2009m:20077 Zbl 1207. 20068

[Sisto 2011] A. Sisto, "Contracting elements and random walks", preprint, 2011. arXiv 1112.2666

[Vogtmann 2002] K. Vogtmann, "Automorphisms of free groups and outer space", Geom. Dedicata 94 (2002), 1-31. MR 2004b:20060 Zbl 1017.20035

Received January 16, 2012. Revised March 20, 2012.

THIERRY COULBOIS

LATP

UNIVERSITÉ D'AIX-MARSEILLE

AVENUE DE L'ESCADRILLE NORMANDIE-NIÉMEN

13013 MARSEILLE

FRANCE

thierry.coulbois@univ-amu.fr

ARNAUd HILION

LATP

Université D'AiX-MARSEILle

AVENUE DE L'ESCADRILLE NORMANDIE-NIÉMEN

13013 MARSEILLE

FRANCE

arnaud.hilion@univ-amu.fr 


\title{
PACIFIC JOURNAL OF MATHEMATICS
}

\author{
http://pacificmath.org \\ Founded in 1951 by \\ E. F. Beckenbach (1906-1982) and F. Wolf (1904-1989)
}

\section{EDITORS}

V. S. Varadarajan (Managing Editor)

Department of Mathematics

University of California

Los Angeles, CA 90095-1555

pacific@math.ucla.edu

Vyjayanthi Chari

Department of Mathematics

University of California

Riverside, CA 92521-0135

chari@math.ucr.edu

\section{Robert Finn}

Department of Mathematics Stanford University

Stanford, CA 94305-2125

finn@math.stanford.edu

Kefeng Liu

Department of Mathematics

University of California

Los Angeles, CA 90095-1555

liu@math.ucla.edu
Darren Long

Department of Mathematics

University of California

Santa Barbara, CA 93106-3080

long@math.ucsb.edu

Jiang-Hua Lu

Department of Mathematics

The University of Hong Kong

Pokfulam Rd., Hong Kong jhlu@maths.hku.hk

Alexander Merkurjev

Department of Mathematics

University of California

Los Angeles, CA 90095-1555

merkurev@math.ucla.edu
Sorin Popa

Department of Mathematics University of California

Los Angeles, CA 90095-1555 popa@math.ucla.edu

Jie Qing

Department of Mathematics

University of California

Santa Cruz, CA 95064

qing@cats.ucsc.edu

Jonathan Rogawski

Department of Mathematics

University of California

Los Angeles, CA 90095-1555

jonr@math.ucla.edu

\section{PRODUCTION}

pacific@math.berkeley.edu

\section{SUPPORTING INSTITUTIONS}

ACADEMIA SINICA, TAIPEI

CALIFORNIA INST. OF TECHNOLOGY INST. DE MATEMÁTICA PURA E APLICADA KEIO UNIVERSITY

MATH. SCIENCES RESEARCH INSTITUTE NEW MEXICO STATE UNIV.

OREGON STATE UNIV.

\author{
STANFORD UNIVERSITY \\ UNIV. OF BRITISH COLUMBIA \\ UNIV. OF CALIFORNIA, BERKELEY \\ UNIV. OF CALIFORNIA, DAVIS \\ UNIV. OF CALIFORNIA, LOS ANGELES \\ UNIV. OF CALIFORNIA, RIVERSIDE \\ UNIV. OF CALIFORNIA, SAN DIEGO \\ UNIV. OF CALIF., SANTA BARBARA
}

\author{
UNIV. OF CALIF., SANTA CRUZ \\ UNIV. OF MONTANA \\ UNIV. OF OREGON \\ UNIV. OF SOUTHERN CALIFORNIA \\ UNIV. OF UTAH \\ UNIV. OF WASHINGTON \\ WASHINGTON STATE UNIVERSITY
}

These supporting institutions contribute to the cost of publication of this Journal, but they are not owners or publishers and have no responsibility for its contents or policies.

See inside back cover or pacificmath.org for submission instructions.

The subscription price for 2012 is US \$420/year for the electronic version, and \$485/year for print and electronic.

Subscriptions, requests for back issues from the last three years and changes of subscribers address should be sent to Pacific Journal of Mathematics, P.O. Box 4163, Berkeley, CA 94704-0163, U.S.A. Prior back issues are obtainable from Periodicals Service Company, 11 Main Street, Germantown, NY 12526-5635. The Pacific Journal of Mathematics is indexed by Mathematical Reviews, Zentralblatt MATH, PASCAL CNRS Index, Referativnyi Zhurnal, Current Mathematical Publications and the Science Citation Index.

The Pacific Journal of Mathematics (ISSN 0030-8730) at the University of California, c/o Department of Mathematics, 969 Evans Hall, Berkeley, CA 94720-3840, is published monthly except July and August. Periodical rate postage paid at Berkeley, CA 94704, and additional mailing offices. POSTMASTER: send address changes to Pacific Journal of Mathematics, P.O. Box 4163, Berkeley, CA 94704-0163.

PJM peer review and production are managed by EditFLOW ${ }^{\mathrm{TM}}$ from Mathematical Sciences Publishers.

PUBLISHED BY PACIFIC JOURNAL OF MATHEMATICS

at the University of California, Berkeley 94720-3840

A NON-PROFIT CORPORATION

Typeset in LATEX

Copyright $(02012$ by Pacific Journal of Mathematics 


\title{
PACIFIC JOURNAL OF MATHEMATICS
}

\author{
Volume $256 \quad$ No. $2 \quad$ April 2012
}

O-operators on associative algebras and associative Yang-Baxter equations

Chengming BAI, Li GuO and Xiang Ni

Botany of irreducible automorphisms of free groups

291

THIERRY COULBOIS and ARNAUd HILION

A note on inverse curvature flows in asymptotically Robertson-Walker 309 spacetimes

Claus GerhardT

Total curvature of graphs after Milnor and Euler

Robert GULLIVER and SUMIO YAMADA

Entire solutions of Donaldson's equation

WEIYONG HE

Energy identity and removable singularities of maps from a Riemann surface with tension field unbounded in $L^{2}$

YONG LUO

Quotients by actions of the derived group of a maximal unipotent subgroup

\section{DMITRI I. PANYUSHEV}

Invariants of totally real Lefschetz fibrations

NERMIN SALEPCI

Stable trace formulas and discrete series multiplicities

STEVEN SPALLONE

Small covers and the Halperin-Carlsson conjecture

\section{YU}

